\title{
Sprechen wir über Kühlschränke? Was die Friedens- und Konfliktforschung von der kritischen entwicklungs- politischen Bildungsarbeit lernen kann
}

Anliegen dieses Beitrags ist es, mein Unbehagen mit der Friedens- und Konfliktforschung zu vermitteln und - ausgehend von meiner persönlichen Erfahrung verschiedene Ursachen dafür aufzuzeigen und zu begründen. Diesen Problematiken stelle ich jeweils Ansätze aus der kritischen entwicklungspolitischen Bildungsarbeit gegenüber, denn meine These ist, dass dadurch »blinde Flecken « der Friedens- und Konfliktforschung erhellt werden können. Dies soll als Anregung dienen, ähnliche Ansätze in die Forschung und Lehre der Friedens- und Konfliktforschung einzubringen. Ich plädiere für eine Selbstreflexion und einen Sensibilisierungsprozess der Friedens- und Konfliktforscher_innen in Bezug auf ihre Position und Rolle in globalen Machtverhältnissen sowie für die Berücksichtigung der damit einhergehenden Konsequenzen.

Schlagworte: (Selbst-)Reflexion der Friedens- und Konfliktforschung, kritische entwicklungspolitische Bildungsarbeit, Rassismus, Kolonialismus

\section{Prolog: Eine Pressemitteilung}

Auch in diesem Jahr kommen rund 50 westafrikanische Wissenschaftler zu einer viertägigen Konferenz in Lagos, Nigeria, zusammen, um das Thema »Psychische Krankheiten bei Kindern in Europa und Nordamerika - gesellschaftliche Ursachen, Wirkungen und Bearbeitungsmöglichkeiten « zu erörtern. Im Fokus stehen diesmal die Themen »Amokläufe an Schulen « sowie»Medikamentenkonsum und-missbrauchals Ausdruck struktureller Gewalt in Gesundheitssystemen «. Viele der hochkarätigen Wissenschaftler gründen ihre Erkenntnisse auf eigene Feldforschungsaufenthalte in Europa und den USA, die ihnen fundierte Einblicke in die gesellschaftlichen Zusammenhänge der diskutierten Problematiken ermöglichen. Ein Merkmal der Konferenz ist somit, dass die Phänomene nicht isoliert betrachtet, sondern in ihrem gesellschaftlichen Entstehungskontext analysiert werden. So wird die strukturelle Gewalt, die in westliche Gesellschaften eingeschrieben ist, deutlich herausgearbeitet und ihre Merkmale wie Effizienzdruck, Konkurrenzdenken, Beschleunigung oder der Fokus auf ökonomische Verwertbarkeit werden als einhergehend mit der Spätmoderne 
analysiert. Dies wiederum dient als Grundlage für die Entwicklung effektiver Bearbeitungskonzepte und-instrumente, die an den Grundursachen ansetzen und nichtnur die Symptome behandeln. Im Rahmen der Konferenz wird hierzu ein sechswöchiges Ausbildungscurriculum für Fachkräfte vorgestellt, welche ab sofort im Rahmen eines Programms der westafrikanischen Union in betroffene Gemeinden in Europa entsandt werden sollen. Des Weiteren wird ein an der Universität Lagos entwickeltes Multi-Stakeholder-Instrument präsentiert, welches Lehrer, Eltern, Behörden und Entscheidungsträger einbezieht, um diese für die Ursachen psychischer Krankheiten bei Kindernzusensibilisieren. Ebenfallswerdenzweineuentwickelte Methodikenzur Wirkungsanalyse von Praxiseinsätzen erstmals einem breiten Publikum vorgestellt. Abgerundet wird die Konferenz von einer Podiumsdiskussion, zu der auch zwei europäische NGO-Vertreterinnen erwartet werden, die sich in ihren Heimatländern für den Schutz der Kindheit einsetzen.

Wie würden wir das finden, wenn Studierende und Wissenschaftler_innen aus Nigeria, Peru oder Myanmar die Abgründe unserer Gesellschaften erforschten - und sich auch noch anmaßten, wissenschaftlich fundierte »Wahrheiten « über uns zutage zu fördern? Ja, wenn sie als Außenstehende womöglich noch zu wissen meinen, wie die Probleme und Konflikte am besten zu bearbeiten sind? Zumindest Unbehagen würde dies bei uns auslösen. Doch eben diese Praxis des Erforschens und (oft ungefragten) Beratens anderer - nur »andersherum « - gehört nach wie vor zum täglichen Geschäft im Kontext der Friedens- und Konfliktforschung. ${ }^{1}$

Das vorangestellte fiktive Szenario ${ }^{2}$ kann einen gefühlsmäßigen Bezug dazu eröffnen, welche Implikationen und Bedeutungen die Friedens- und Konfliktforschung mit ihren Handlungs- und Ausdrucksweisen mit sich bringen kann. Ich vermute, dass es Sie in einer oder mehrerer Hinsicht gefühlsmäßig berührt hat und es vielleicht sogar ganz spontan neue Sichtweisen oder gar einen Aha-Effekt bei Ihnen auslösen konnte. Vielleicht hat es Sie angeregt, das, was Sie in Ihrem Studium, Ihrer Forschung oder Ihrer Lehre normalerweise tun, kritisch zu hinterfragen und aus einer anderen Perspektive zu betrachten.

1 Der Text basiert auf einem Beitrag zur dritten Konferenz junger Wissenschaftler_innen der Arbeitsgemeinschaft für Friedens- und Konfliktforschung (AFK)»What the FuK?! Kritische Perspektiven in der/auf die Friedens- und Konfliktforschung«, die vom 2. bis 3. April 2014 in Schwerte-Villigst stattfand. Ich danke Michaela Zöhrer und Teresa Koloma Beck für konstruktive Kritik und Anregungen.

2 Die Idee eines Szenarios mit »vertauschten Rollen« stammt von Chandra-Milena Danielzik und Beate Flechtker (2012). 


\section{Anliegen und »Methode $«$}

In diesem Beitrag möchte ich - ausgehend von meiner persönlichen Erfahrung mein Unbehagen mit der Friedens- und Konfliktforschung vermitteln. Dieses kenne ich aus meinem Studium, aus der Auseinandersetzung mit Forschung sowie von Konferenzen und anderen Formen des Austauschs der wissenschaftlichen Community. Diesem Unbehagen möchte ich wertvolle Lernerfahrungen aus der kritischen entwicklungspolitischen Bildungsarbeit gegenüberstellen, denn meine These ist, dass die »blinden Flecken« des ersteren Kontextes durch Ansätze des letzteren erhellt werden können.

Seit 2005 bewege ich mich in Kontexten der Friedens- und Konfliktforschung, der Praxis der zivilen Konfliktbearbeitung bzw. Friedensarbeit sowie der entwicklungspolitischen Bildungsarbeit. Ich habe mich mit den jeweiligen Themen, Diskursen und Arbeitsweisen auseinandergesetzt und diese immer auch kritisch beobachtet. Dabei habe ich in jedem dieser Kontexte viel Wertvolles gelernt, jedoch auch deutliches Unbehagen empfunden bezüglich bestimmter Arten und Weisen zu denken, zu sprechen und zu handeln, die mir für den jeweiligen Kontext charakteristisch erscheinen.

Mein Beitrag lässt mein Unbehagen mit der kritischen entwicklungspolitischen Bildungsarbeit sowie meine wertvollen Lernerfahrungen aus der Friedens- und Konfliktforschung unberücksichtigt - die es jedoch gleichermaßen gibt. Mit anderen Worten: Auch die Frage, inwiefern und was die entwicklungspolitische Bildungsarbeit von der Friedens- und Konfliktforschung lernen kann, wäre eine legitime und interessante Frage, deren Beantwortung im Weiteren jedoch nicht verfolgt wird.

Auch beansprucht mein Beitrag nicht, Aussagen über »die« Friedens- und Konfliktforschung als solche zu treffen. ${ }^{3}$ Meine Argumentation geht von meiner persönlichen, partikularen Erfahrung aus, die ich jedoch auf eine Weise reflektiere, dass sie, so meine ich, über das Partikulare hinausweisen und Denkanstöße und Hinweise - nicht aber Aussagen oder Bewertungen - für »die« Friedens- und Konfliktforschung als solche geben kann. ${ }^{4}$

Wenn ich in diesem Beitrag - ganz bewusst - von »wir« spreche, wende ich mich an eine imaginierte Leserschaft von weißen, westlich sozialisierten Wissenschaft-

3 Ebenso wenig über »die« entwicklungspolitische Bildungsarbeit als solche.

4 Meine für die Friedens- und Konfliktforschung relevantesten Erfahrungskontexte waren mein Masterstudium an der Universität Marburg (2005-2008) sowie die Teilnahme an AFK-Kolloquien und anderen einschlägigen Tagungen. Im Hinblick auf die kritische entwicklungspolitische Bildungsarbeit haben mich insbesondere meine Erfahrungen im Rahmen des ASA-Programms als Teilnehmerin, Trainerin und Ehrenamtliche (2006-2013) geprägt. Verschiedene weitere Erfahrungskontexte in den genannten Bereichen ergänzen meine Eindrücke. 
ler_innen, durch die die deutsche Friedens- und Konfliktforschung ja auch maßgeblich geprägt ist, und schließe mich bewusst mit ein. Das »Wir« möge hier mehr als sprachliches Mittel gelesen werden denn als unberechtigte Vereinnahmung einer immer vielfältiger werdenden Disziplin.

So möchte ich im Folgenden verschiedene Ursachen für mein genanntes Unbehagen mit der Friedens- und Konfliktforschung darlegen und begründen. Daran werde ich jeweils Ansätze aus der entwicklungspolitischen Bildungsarbeit des ASA-Programms ${ }^{5}$ anschließen, die der aufgeworfenen Problematik konstruktiv begegnen. Dies soll als Anregung dienen, ähnliche Ansätze in die Forschung und Lehre der Friedens- und Konfliktforschung einzubringen.

Das ASA-Programm versteht sich als »entwicklungspolitische Lernwerkstatt" und bemüht sich fortlaufend um kritische, macht- und rassismussensible Ansätze. Für die Teilnehmenden steht oft zunächst ein mehrmonatiges entwicklungspolitisches Praktikum in Asien, Afrika, Lateinamerika oder Südosteuropa im Mittelpunkt des Interesses. Einen hohen Stellenwert hat im Gesamtkonzept des Programms jedoch auch das begleitende Seminarprogramm, welches die Teilnehmenden in ihrem Engagement für eine »nachhaltige und global gerechte Welt« (ASA-Programm 2014) fördern möchte. Es dient gleichzeitig zur Vor- und Nachbereitung des Auslandsaufenthaltes, wobei der Fokus hierbei auf einer Sensibilisierung und der Vermittlung einer kritischen Sichtweise liegt. Für viele Teilnehmende schließt sich ein Engagement als Ehrenamtliche oder Trainer_innen im ASA-Netzwerk an, wodurch die Auseinandersetzung mit globalen Themen auf vielfältige Weise vertieft werden kann. ${ }^{6}$

Eine Herausforderung bei der Frage, was die Friedens- und Konfliktforschung von der kritischen entwicklungspolitischen Bildungsarbeit lernen kann, ist die Gegenüberstellung einer wissenschaftlichen Disziplin mit einem (nichtwissenschaftlichen) Praxisfeld. Dies impliziert, dass Ansätze, Methoden und Quellen, die in diesem Feld und auch im ASA-Programm zur Anwendung kommen, nicht den Kriterien wissenschaftlichen Materials entsprechen. Dieser Umstand macht die Ansätze, Methoden und Quellen jedoch nicht ungeeignet für die Friedens- und Konflikt-

5 Die Bildungsarbeit des ASA-Programms dient hier deshalb als Quelle, da ich diesbezüglich auf umfassende persönliche Erfahrungen zurückgreifen kann.

6 In einem Auszug aus der Selbstbeschreibung des ASA-Programms heißt es: »Das Besondere am ASA-Programm ist das Zusammenspiel von Trainingsseminaren in Deutschland und Europa sowie einem drei- bis sechsmonatigen Projektpraktikum in einem von mehr als 50 Ländern in Afrika, Asien, Lateinamerika oder Südosteuropa. Im Anschluss an das Projektpraktikum werden die Erfahrungen gemeinsam ausgewertet und Methoden und Möglichkeiten für ein weiteres Engagement in der entwicklungspolitischen Bildungsarbeit in Deutschland und Europa vermittelt« (siehe hierzu: http:// www.asa-programm.de/ueber-asa/das-asa-programm.html, letzter Zugriff: 24.9.2014). 
forschung, denn ich plädiere hier nicht notwendigerweise für eine wissenschaftliche Auseinandersetzung mit den aufgeführten Problematiken, sondern für eine Bewusstwerdung und Sensibilisierung, mit der sich Friedens- und Konfliktforscher_innen verantwortungsvoller ihren jeweiligen Themen und Tätigkeiten widmen können. Diese Sensibilisierungserfahrung kann vielleicht sogar leichter durch Bildungsmaterialien zugänglich sein als durch wissenschaftliche Abhandlungen.

\section{Sprechen wir über Kühlschränke? - Machtposition und Perspektivenwechsel}

In der Friedens- und Konfliktforschung habe ich wenig Auseinandersetzung mit unserer eigenen Machtposition als weiße, westliche Forscher_innen erlebt. Die Frage, was das Sprechen über und für Andere bedeuten und bewirken kann, wird noch viel zu selten gestellt.

Ein Beispiel: Auf einer Konferenz zu ziviler Krisenprävention wurden Fragen zur »Förderung von Zivilgesellschaft und Menschenrechten« in einer Arbeitsgruppe erörtert. Es wurde dabei über Menschen und Gesellschaften in »Konfliktregionen« auf eine Weise gesprochen, die mir Unbehagen bereitete. Ich fragte mich, was genau dieses Unbehagen ausmachte und hatte plötzlich ein Bild vor Augen: Auf die gleiche Weise könnte sich eine Arbeitsgruppe auf einer Konferenz der Hausgeräte-Industrie über Kühlschränke unterhalten!

Das Sprechen über ferne Wirklichkeiten und Menschen anderer Gesellschaften als »Forschungsgegenstände« oder »Zielgruppen« macht diese (zumindest in unserem Diskurs und unserem Denken) schnell zu unmündigen Objekten. Denn wenn wir uns selbst genau beobachten, dann scheint mir, dass bei diesem ganzen Denken, Sprechen und Schreiben über »Konfliktregionen « - oder auch »die lokale Bevölkerung « - das Gefühl, d. h. der wirklich fühlende Bezug dazu verloren geht, dass wir es hierbei mit Menschen zu tun haben, die fühlen, erleben, wollen und handeln - und ja, die für sich selbst denken, sprechen und schreiben! Anders als Kühlschränke.

Ein erster Schritt, um der Verantwortung unserer Machtposition im Sprechen und Schreiben gerecht zu werden, besteht darin, sich dieser Mechanismen und Gewohnheiten überhaupt erst einmal bewusst zu werden. Dies kann durch einen bewusst eingesetzten Perspektivenwechsel angeregt werden, der ermöglicht, die Position der »Zielgruppen« gedanklich einzunehmen und so die Wirkungen des eigenen Tuns zu erahnen. Ein Beispiel hierfür stellt das Szenario am Anfang dieses Beitrags dar.

Perspektivenwechsel einzuüben und als Methode kritischer Reflexion zu nutzen, ist ein wichtiges Anliegen der Bildungsarbeit des ASA-Programms. Dabei wird insbesondere darauf geachtet, die sogenannte »Südperspektive« bei möglichst 
vielen Themen und Fragestellungen einzubringen und mitzudenken. Dies bedeutet, sowohl sich selbst gedanklich in die Position von Menschen aus Ländern des Südens hineinzuversetzen als auch Menschen aus diesen Regionen selbst zu Wort kommen zu lassen und als aktiv Beitragende und Teilnehmende ins Programm miteinzubeziehen. Diese Herangehensweise kann auf die Friedens- und Konfliktforschung übertragen werden und dort für die Lehre wie auch für Forschung und Wissenschaftsbetrieb nutzbar gemacht werden.

\section{Start with firstly - Kolonialismus mitdenken}

In der Friedens- und Konfliktforschung habe ich wenig Reflexion über Kolonialismus und koloniales Erbe wahrgenommen - und das, obwohl die Inhalte der Friedens- und Konfliktforschung diese Fragen regelrecht implizieren. Um diese Implikationen - und damit die Notwendigkeit einer solchen Reflexion - zu illustrieren, möchte ich Chimamanda Ngozi Adichie (2009) zitieren:

»The Palestinian poet Mourid Barghouti writes that if you want to dispossess a people, the simplest way to do it is to tell their story and to start with >secondly . Start the story with the arrows of the Native Americans, and not with the arrival of the British, and you have an entirely different story. Start the story with the failure of the African state, and not with the colonial creation of the African state, and you have an entirely different story « (Adichie 2009: Absatz 10-11).

Dieses Zitat macht deutlich, wie viel die Einbeziehung oder Nicht-Einbeziehung von Kolonialgeschichte für die Analyse von Krieg und Frieden ausmachen kann. Damit möchte ich nicht vorwegnehmen, wie solche Analysen auszufallen haben jedoch kann Kolonialgeschichte kaum angemessen einbezogen oder zumindest kritisch geprüft und mitgedacht werden, wenn das Wissen über und das Bewusstsein für diese fehlt, wenn also Studierende und Wissenschaftler_innen nicht anfangen, sich mit diesen Themen offen und neugierig auseinanderzusetzen.

Das Zitat von Chimamanda Ngozi Adichie ist Teil einer Rede mit dem Titel »The danger of a single story«, die zur Pflichtlektüre aller Teilnehmer_innen des ASAProgramms gehört. Mit dieser und ähnlich augenöffnenden Quellen könnte sich auch in der Friedens- und Konfliktforschung auseinandergesetzt werden. ${ }^{7}$

7 Hinweise für weitere (nichtwissenschaftliche) Quellen bieten die Broschüren des Berliner Entwicklungspolitischen Ratschlags (2012; 2013). 


\section{Was hat Rassismus mit mir zu tun?}

Auch habe ich in der Friedens- und Konfliktforschung nicht gelernt, dass Rassismus etwas mit mir zu tun hat. Dass Rassismus also nicht etwas ist, was sich nur auf NeoNazis und Rechtsextremismus beschränkt, sondern eine Machtstruktur darstellt, die die deutsche Gesellschaft, ja die globale Gesellschaft durchzieht und somit gerade für Friedens- und Konfliktforscher_innen relevant ist. Zum einen ist es wichtig, diese Machtstruktur als eine Form von struktureller Gewalt zu erkennen, da dies uns ermöglicht, die rassistischen Dimensionen von Konflikten in unseren Analysen zu berücksichtigen. Zum anderen aber macht dieses Rassismus-Verständnis einen zentralen Unterschied für Friedens- und Konfliktforscher_innen, da diese globale Machtstruktur uns selbst einschließt, während wir uns mit globalen Themen befassen und ggf. global und interkulturell interagieren (z. B. im Rahmen von Auslandspraktika oder Feldforschungsaufenthalten).

Somit ist es von Bedeutung, ob wir unsere eigene Rolle und Position in dieser Machtstruktur erkennen und verstehen und damit unseren eigenen, in der Regel nicht-intendierten und unbewussten Rassismus entdecken. Es ist natürlich nicht gerade angenehm, seine eigenen Denk- und Handlungsweisen als rassistisch zu entlarven - selbst wenn man sich darüber im Klaren ist, dass dies strukturelle Ursachen hat und sogar gegen die besten Absichten resistent ist (vgl. Weiß 2001). Die Kunst besteht hier - so finde ich - darin, sich selbst nicht dafür zu verurteilen, wenn man eigene rassistische Denkweisen bei sich erkennt, sondern im Gegenteil, dies als Weiterentwicklung einzuordnen.

Dieses Erkennen wurde bei mir durch das ASA-Seminarprogramm angestoBen. ${ }^{8}$ Die Ziele der ASA-Seminare untergliedern sich in fünf inhaltliche Säulen, von denen wohl diejenige zum Thema »Reflexion von Machtstrukturen« die prominenteste ist und die größte Aufmerksamkeit, Auseinandersetzung und Weiterentwicklung erfährt. ${ }^{9}$ In den Zielen der ASA-Seminare wird das wie folgt begründet und inhaltlich ausgeführt:

8 In den letzten Jahren ist das ASA-Programm in die Kritik geraten, als Organisation selbst von strukturellem Rassismus betroffen zu sein. Dies steht für mich jedoch nicht im Widerspruch dazu, Lernerfahrungen aus dem ASA-Kontext für die Friedens- und Konfliktforschung abzuleiten. Vielmehr zeigt diese Tatsache, dass selbst Organisationen, in denen starke Motivationen und Bestrebungen vorhanden sind, sich und andere für Rassismus zu sensibilisieren, sich nicht leicht der Wirkmacht dieser globalen Machtstruktur entziehen können.

9 Die weiteren Ziele heißen: Vorbereitung der Praxisphase, Globale Zusammenhänge, Vernetzung und Engagement, Lernprozesse. 
»Das ASA-Programm ist im entwicklungspolitischen Kontext verortet und die Praxisphase findet in einem Land des globalen Südens (oftmals mit einer Kolonialgeschichte) statt. Insbesondere in diesem Nord-Süd-Kontext, mit seinen kolonialen Kontinuitäten, ist die Auseinandersetzung mit (globalen) Machtstrukturen von zentraler Relevanz. Die Teilnehmenden sollen sich daher im Kontext der Seminare mit individuellem und strukturellem Rassismus, Kolonialismus und Entwicklungspolitik in diesem Kontext auseinandersetzen. Während für die weißen Teilnehmenden hier eine kritische Selbstreflexion von Privilegien im Vordergrund steht, sollten den POC-positionierten Teilnehmenden empowernde Angebote zum Umgang mit Rassismus gemacht werden« (ASA-Programm 2014). ${ }^{10}$

Diese Argumentation lässt sich auch auf die Friedens- und Konfliktforschung übertragen, da sich die überwiegende Zahl der Konfliktkontexte, die sie betrachtet, in Ländern des »globalen Südens« befinden. So könnte hier eine ähnliche Konsequenz gezogen werden, sich z. B. durch Trainingsangebote mit diesen Machtstrukturen auseinanderzusetzen und bereits im Studium dafür zu sensibilisieren. ${ }^{11}$ Selbstverständlich kann dies durch eine wissenschaftliche Auseinandersetzung mit den Themenkomplexen Kolonialismus und Rassismus ergänzt und vertieft werden.

\section{Will ich überhaupt weg? - Feldforschung in Ländern des Südens}

Mit Vorliebe wird in der Friedens- und Konfliktforschung der Blick weit in die Ferne gerichtet - Feldforschung in Afrika, Asien oder Lateinamerika ist en vogue. Studierende werden zu Feldforschungsaufenthalten und Freiwilligendiensten in konfliktiven Regionen und Nachkriegsgesellschaften ermutigt und angeleitet (BuckleyZistel 2012; Koltermann 2013). Begründet wird dies damit, dass Friedens- und Konfliktforscher_innen auf diese Weise ein besseres und tieferes Verständnis von Frieden und Konflikten gewinnen können, insbesondere »im Sinne von Gespür für das Leben in unsicheren Gefilden und die Auswirkungen von Krieg und Gewalt auf die betroffenen Menschen« (Buckley-Zistel 2012: 315). Dieser Einschätzung stimme ich zu, jedoch ist ein solches Unterfangen eine große Herausforderung, ja meist eine Überforderung für die Forscher_innen, und dies nicht nur, weil es, wie Felix Koltermann (2013) ausführt, gleichzeitig hohe Anforderungen an die fachliche, soziale, emotionale und (selbst-)organisatorische Kompetenz der Forschenden stellt

$10 \mathrm{POC}=$ Person of color (Plural: people of color) ist eine politische Selbstbezeichnung von Personen, die sich Rassismus ausgesetzt und dadurch von der Mehrheitsgesellschaft ausgegrenzt fühlen.

11 Auch hier bieten die Broschüren des Berliner Entwicklungspolitischen Ratschlags (2012; 2013) weiterführende Textmaterialien und Hinweise. 
sowie Gefahren für Gesundheit und Sicherheit mit sich bringt. Sondern zusätzlich, weil ein verantwortungsvoller Feldaufenthalt meiner Meinung nach einen ernsthaften Sensibilisierungsprozess in Bezug auf Machtasymmetrien, rassistische und postkoloniale Strukturen sowie die eigene Rolle und Position darin voraussetzt. Dieser Sensibilisierungsprozess kann dann während des Feldaufenthalts als Orientierungs- und Interpretationsfolie dienen, um irritierende soziale Erfahrungen einordnen und auf machtkritische und selbstreflexive Weise verstehen zu können. Nur so besteht überhaupt die Chance, mit sozialen Interaktionen »im Feld « sensibel und verantwortungsvoll umzugehen.

Wie oben beschrieben, leistet das Seminarprogramm von ASA eine solche Sensibilisierung im Vorfeld der Auslandsaufenthalte durch die Themensäule »Reflexion von Machtstrukturen«. Dabei wird auch ganz explizit der Sinn entwicklungspolitischer Auslandsaufenthalte kritisch hinterfragt und deren vielfältige Implikationen den Teilnehmer_innen bewusst gemacht. Dies führt nur selten dazu, den geplanten Auslandsaufenthalt abzusagen. Jedoch verlagern viele, die sich längerfristig im ASA-Programm engagieren und kontinuierlich mit diesen Fragen befassen, den Fokus ihres (entwicklungspolitischen) Engagements auf ihre eigene Gesellschaft. ${ }^{12}$

Darüber hinaus wird ein verantwortungsvoller und rassismuskritischer Umgang mit Bildern und Sprache vermittelt. Dies hat insofern Bedeutung, da Auslandsaufenthalte eine Erfahrungskategorie sind, die den Daheimgebliebenen gerne durch geschriebenes und gesprochenes Wort sowie durch Fotos vermittelt wird und es dabei häufig ungewollt zu rassistischen und stereotypen Darstellungen kommt (glokal e. V. 2012). Daher sollte gerade auch Feldforschung in Ländern des Südens im Hinblick auf diese Thematik vorbereitet werden, zumal sie sich in der Regel in geschriebenen (und publizierten) Texten niederschlägt. ${ }^{13}$

\section{Blinde Flecken - Verwobenheit in globale Konfliktdynamiken}

Weiterhin erscheint mir der oft selbstverständliche und unhinterfragte Fokus auf »ferne Wirklichkeiten« in der Friedens- und Konfliktforschung als eine Art blinder Fleck im Sinne eines (unbewussten) Wegschauens von sich selbst und der eigenen Gesellschaft. Damit bleibt die eigene Rolle (als Individuum und als Gesellschaft) für die Reproduktion globaler Ungleichheit sowie für globale Umwelt- und Ressourcenprobleme und somit für globale Konfliktursachen unbeleuchtet.

12 Dies wird beispielsweise deutlich bei den von aktiven Ehrenamtlichen gewählten Jahresthemen wie »Entwicklungsland Deutschland «, »Entschleunigung « oder »Degrowth«.

13 Erste Einblicke in dieses Thema geben glokal e. V. (2012) und Wainaina (2005). 
Die Auseinandersetzung mit globalen Zusammenhängen sowie mit der eigenen Rolle darin ist demgegenüber eine weitere wichtige Säule der Bildungsarbeit des ASA-Programms (ASA-Programm 2014). Es werden dabei verschiedene ökologische, politische und soziale Themen bearbeitet, z. B. Klimawandel und ökologischer Fußabdruck, Migration, Weltwirtschaftsbeziehungen, Konsum und Wirtschaftswachstum. Es wird den Teilnehmenden dabei z. B. bewusst, was auf der Welt passiert, damit sie eine Jeans oder ein Handy kaufen können. Oder wie groß ihr ökologischer Fußabdruck ist und was dies mit dem Klimawandel zu tun hat, der vor allem in »Ländern des Südens « mit seinen Auswirkungen zum Tragen kommt.

Würden in der Friedens- und Konfliktforschung die Zusammenhänge und Auswirkungen unseres Lebensstils und unserer politischen und gesellschaftlichen Verhältnisse im globalen Kontext systematisch analysiert, müssten wir uns als Teil vieler Konfliktdynamiken erkennen. Während wir dies jedoch nicht bewusst mitdenken, formt sich unser Selbstbild als »Friedensexpert_innen « und wir können »die Guten « sein, ohne uns mit uns selbst zu konfrontieren. Ich glaube, dass dieses Selbstbild und Selbstverständnis sich ganz unbemerkt und unbewusst »einschleicht«, während man mit den besten, vielleicht sogar mit idealistischen Absichten Friedens- und Konfliktforschung betreibt. Selbst wenn man bewusst weder normativ noch politisch motiviert forscht und arbeitet, schwingt dieses Selbstverständnis, so glaube ich, als eine Art tiefenkulturelle Grundannahme mit.

Ich habe ein solches Selbstbild bei mir entdeckt. Vielleicht entdecken Sie es auch bei sich? Während Bettina Engels vorschlägt, »die Selbst-Bilder der [...] Beteiligten von Interventionen der Konfliktbearbeitung und Friedensförderung « in den Blick zu nehmen, da Studien gezeigt hätten, wie Akteure »in Entwicklungszusammenarbeit und Humanitärer Hilfe eigene Identitäten eines `humanitär` und >partnerschaftlich ' helfenden Selbst konstruieren « (Engels 2014: 138), äußere ich hier die Vermutung, dass ein ähnliches Selbstbild auch bei Wissenschaftler_innen wirkmächtig ist. Daher schlage ich vor, dass Friedens- und Konfliktforscher_innen nicht nur die Verwobenheit ihrer Selbst und ihrer Gesellschaft mit Konfliktdynamiken im globalen Kontext wahrnehmen und in ihrer inhaltlichen Arbeit berücksichtigen, sondern auch ihr eigenes Selbstbild in den Blick rücken. Ziel dessen ist nicht eine Selbstanklage, sondern zu verhindern, dass das Gefühl, »zu den Guten zu gehören «, unbewusst wie eine Erlaubnis wirkt, sich selbst und die eigene Arbeit nicht kritisch hinterfragen zu müssen. 


\section{Epilog: Und nun?}

Sich all den aufgeworfenen Fragen zu stellen - auf einer allgemeinen wie auch auf einer ganz persönlichen Ebene - sollte meiner Meinung nach integraler Bestandteil der friedens- und konfliktwissenschaftlichen Studiengänge sowie des Selbstverständnisses der Forschungs-Community sein. Dabei geht es nicht darum, sich einer ideologischen Norm zu verschreiben oder einfache Antworten zu finden. Vielmehr geht es mir um eine ergebnisoffene Erkundung dieser Fragen sowie um einen Sensibilisierungsprozess der Friedens- und Konfliktforscher_innen in Bezug auf ihre Position und Rolle in globalen Machtverhältnissen. Sich dem unerschrocken zu stellen, würde die Friedens- und Konfliktforschung, die für sich den Anspruch erhebt, potenziell jeden Konflikt auf der Welt erforschen zu können (und dabei friedensfördernd zu wirken), sich selbst näher bringen.

\section{Literatur}

Adichie, Chimamanda Ngozi 2009: The danger of a single story (Vortrag, TED conference), in: http://www.ted.com/talks/chimamanda_adichie_the_danger_ of_a_single_story/transcript; 3.9.2014.

ASA-Programm 2014: Ziele der ASA-Seminare 2014 (internes Dokument), Berlin. Berliner Entwicklungspolitischer Ratschlag (Hrsg.) 2012: Wer andern einen Brunnen gräbt. Rassismus//Empowerment//Globaler Kontext (Broschüre), Berlin.

Berliner Entwicklungspolitischer Ratschlag (Hrsg.) 2013: Develop-mental Turn. Neue Beiträge zu einer rassismuskritischen entwicklungspolitischen Bildungsund Projektarbeit (Broschüre), Berlin.

Buckley-Zistel, Susanne 2012: »Ich bin dann mal weg«. Feldforschung im Studium der Friedens- und Konfliktforschung, in: Zeitschrift für Friedens- und Konfliktforschung 1:2, 315-323.

Danielzik, Chandra-Milena/Flechtker, Beate 2012: Wer mit Zweitens anfängt. Bildung für nachhaltige Entwicklung kann Machtwissen tradieren, in: iz3w Nr. 329, D8-D10.

Engels, Bettina 2014: Repräsentationen, Diskurse und Machtfragen: Postkoloniale Theorieansätze in der Friedens- und Konfliktforschung, in: Zeitschrift für Friedens- und Konfliktforschung, 3: 1, 130-150.

glokal e. V. (Hrsg.) 2012: Mit kolonialen Grüßen... Berichte und Erzählungen von Auslandsaufenthalten rassismuskritisch betrachtet (Broschüre), Berlin. 
Koltermann, Felix 2013: Vom Hier und Dort - Lernen für die Praxis der Feldforschung. Eine Replik auf Susanne Buckley-Zistel, in: Zeitschrift für Friedensund Konfliktforschung 2: 1, 130-140.

Wainaina, Binyavanga 2005: How to write about Africa, in: Granta No. 92, 91-95.

Weiß, Anja 2001: Rassismus wider Willen. Ein anderer Blick auf eine Struktur sozialer Ungleichheit, Opladen.

\section{Die Autorin}

Vera Kahlenberg ist wissenschaftliche Mitarbeiterin an der Forschungseinheit für Sozioökonomie der Arbeits- und Berufswelt der Universität Augsburg.

E-Mail: vera.kahlenberg@phil.uni-augsburg.de 